\title{
Enseñanza de la iniciación a la traducción basada en competencias: resultados de una validación empírica
}

\author{
Anabel Galán-Mañas \\ Universitat Autònoma de Barcelona \\ isabel.galan@uab.cat
}

\section{Resumen}

En este artículo presentamos un estudio empírico con el objetivo de evaluar la validez de una propuesta pedagógica para la formación inicial del traductor basada en competencias. Damos alguna pincelada sobre el marco conceptual de la propuesta pedagógica y detallamos las competencias de aprendizaje, los contenidos, la secuenciación de las unidades didácticas y el sistema de evaluación. Por último, se presenta la experimentación de la propuesta: contexto, instrumentos de recogida de datos y resultados obtenidos, que demuestran la idoneidad de la propuesta y ponen de manifiesto que la semipresencialidad promueve la autonomía del estudiante y fomenta el trabajo en grupo.

Palabras-clave: Enseñanza de la traducción, iniciación a la traducción, formación por competencias, enfoque por tareas, evaluación, semipresencialidad.

\section{Resumo}

Neste artigo apresentamos um estudo empírico com o objetivo de avaliar a validade de uma proposta pedagógica para a formação inicial do tradutor baseada em competências. Explicamos o marco conceitual de proposta pedagógica e damos detalhes sobre as competências de aprendizagem, os conteúdos, a sequência das unidades didáticas e o sistema de avaliação. Por ultimo, apresentamos o estudo empírico: contexto, instrumentos usados para recolher dados e os resultados obtidos. Estes resultados demonstram a idoneidade da proposta e evidenciam que o blended learning promove a autonomia do estudante e fomenta o trabalho em grupo.

Palavras-chave: Ensino da tradução, iniciação à tradução, formação por competências, enfoque por tarefas, blended learning. 


\section{Abstract}

In this paper I present an empirical study to evaluate the validity of a pedagogical proposal for competence-based translator training. I explain the conceptual framework of the pedagogical proposal, as well as the learning competencies, content, sequencing of the didactic units and the assessment system. Finally, I show the context, the data collection instruments and the results of the research, which demonstrate the suitability of the proposal and show that blended learning promotes student autonomy and fosters teamwork. Keywords: Translation training, introduction to translation, competencebased training, task-based approach, assessment, blended learning.

\section{Introducción}

De acuerdo con las directrices del Espacio Europeo de Educación Superior, las propuestas pedagógicas para la enseñanza universitaria deben insertarse en la formación por competencias. El término competencia, como indican Zabala y Arnau (2007, p. 19) surgió del ámbito de la empresa para designar la capacidad o habilidad de efectuar tareas o hacer frente a situaciones diversas de forma eficaz en un contexto determinado y para ello es necesario movilizar actitudes, habilidades y conocimientos al mismo tiempo y de forma interrelacionada.

En el caso de la enseñanza de la traducción, entendemos que para que el estudiante alcance competencia traductora, debe adquirir los conocimientos y las habilidades necesarios para traducir. En ese sentido, para enseñar a traducir tomamos como punto de partida la definición de competencia traductora propuesta por el grupo PACTE (2005) y su distinción en subcompetencias bilingüe, extralingüística, de conocimientos en traducción, instrumental y estratégica.

Para ayudar al estudiante a desarrollar la competencia traductora y, siguiendo una línea constructivista, hemos adoptado el enfoque por tareas, introducido en la enseñanza de la traducción por Hurtado (1992). Este enfoque dota al diseño curricular de un carácter globalizador capaz de integrar todos sus elementos - competencias, contenidos de aprendizaje, metodología y evaluación-, al tiempo que ayuda al individuo a construir sus propios conocimientos, y no a memorizar las respuestas "correctas". 
Partiendo de estos presupuestos pedagógicos, en este artículo presentamos una propuesta pedagógica basada en competencias para la formación inicial del traductor y los resultados de su experimentación. Explicaremos el marco conceptual que articula la propuesta, detallaremos los elementos que la articulan $\mathrm{y}$, por último, presentaremos los resultados de un estudio empírico realizado con el objetivo de analizar la idoneidad de la propuesta.

\section{Marco conceptual}

\subsection{Presupuestos pedagógicos}

Para articular el proceso de enseñanza y aprendizaje seguimos un modelo constructivista. Este modelo, impulsado por Piaget (1967), defiende que los estudiantes construyen sus aprendizajes a partir de aprendizajes anteriores. En consecuencia, el aprendiz va enlazando sus antiguos conocimientos con los nuevos y así va adquiriendo sus competencias. El autor también denominó su teoría aprendizaje por acción, dado que, según él, el estudiante aprende haciendo, actuando. Aprender haciendo es clave para aprender a traducir, debido a que la competencia traductora es una competencia básicamente procedimental.

El enfoque por tareas se adapta a este modelo constructivista porque el estudiante debe ir ejecutando tareas que progresivamente adquieren mayor dificultad. Este enfoque, diseñado para la enseñanza de lenguas, fue introducido en la enseñanza de la traducción por Hurtado (1996 y 1999), que define tarea como una unidad de trabajo en el aula, representativa de la práctica traductora, que se dirige intencionalmente al aprendizaje de la traducción y que está diseñada con un objetivo concreto, una estructura y una secuencia de trabajo (1999: 48). Posteriormente, otros autores han presentado diferentes propuestas pedagógicas para la enseñanza de la traducción de diferentes combinaciones lingüísticas basadas en el enfoque por tareas (Díaz Fouces, 1999; Sevilla, 2002; Orozco, 2007; Gamero, 2005; González Davis, 2003 y 2004). La gran aceptación del enfoque por tareas en sus diferentes campos de aplicación se debe a los propios 
principios en que se asienta dicho enfoque, entendiendo que para el aprendizaje es más importante el proceso que el producto, y que las tareas integran todos los elementos del diseño curricular: objetivos de aprendizaje y competencias, contenidos de aprendizaje, evaluación, papel del profesorado y del estudiantado.

\subsection{Formación por competencias}

Para definir competencia, tomamos la definición de Zabala y Arnau (2008: 43), que apuntan que es la capacidad o habilidad de efectuar tareas o hacer frente a situaciones diversas de forma eficaz en un contexto determinado y para ello es necesario movilizar actitudes, habilidades $y$ conocimientos al mismo tiempo $y$ de forma interrelacionada.

La importancia de la enseñanza por competencias en el ámbito académico reside en que al ser un modelo aplicado en los contextos profesionales, las empresas valoran muy positivamente a los graduados que se han formado por competencias, ya que ello asegura que el individuo no sólo sabe, sino que también sabe hacer.

Existen competencias genéricas y competencias específicas. Las genéricas, también llamadas transversales, se pueden transferir a diferentes contextos, ámbitos profesionales y tareas. Las específicas son las propias de una titulación o profesión.

Para llevar a cabo una formación por competencias, según sostienen Villa y Poblete (2007: 52), hay que cumplir los siguientes criterios: determinar claramente en qué consiste la competencia que se desea desarrollar y evaluar; formular explícitamente el propósito que se pretende en cada actividad; señalar el contexto en que se van a realizar las actividades, estimar el tiempo aproximado en que deberían realizarse las actividades; indicar los materiales o recursos necesarios, bien sean dados o que deba buscarlos el estudiante; señalar la estrategia (métodos y procedimientos) para llevar a cabo la acción prevista; ofrecer indicadores de lo que se entiende que es una adecuada demostración de la competencia (criterios de evaluación relacionados con los indicadores y evidencias).

En la formación de traductores, pocos autores han tratado las competencias (Kelly, 2005; y Hurtado 2007, 2008; Galán-Mañas, 2007 y 2009; Calvo, 2011; Andújar y Cañada, 2011). Kelly (2005) 
reflexiona sobre todos los elementos que debe tener en cuenta el didacta a la hora de elaborar un diseño curricular. Por otro lado, Hurtado (2007 y 2008) establece las bases para una planificación curricular basada en competencias para la formación de traductores y propone unas categorías de competencias específicas de traducción para las materias relacionadas directamente con la formación de traductores, es decir, la iniciación a la traducción hacia la lengua materna, la traducción hacia la lengua extranjera y las diferentes ramas de especialización (traducción técnica, científica, jurídica, audiovisual, literaria, localización, etc.). Dichas categorías son: metodológicas, estratégicas, contrastivas, extralingüísticas, profesionales, instrumentales y textuales (2008: 12). Galán-Mañas (2007) presenta una propuesta pedagógica para la enseñanza de la traducción basada en objetivos de aprendizaje y competencias y en 2009 y 2010 publica los resultados de una validación empírica de varias propuestas pedagógicas para la enseñanza de la traducción general y especializada en diferentes combinaciones lingüísticas y en la modalidad semipresencial. Calvo (2011) se centra en la aplicación de un modelo de competencia traductora al diseño curricular universitario. Andújar y Cañada (2011) presentan una propuesta didáctica centrada en la traducción jurídica francés-castellano, donde el trabajo por competencias y el fomento de la autonomía del aprendiz desempeñan un papel preponderante

La evaluación es un elemento clave en cualquier diseño curricular, por un lado dado el impacto que tiene en el aprendizaje del estudiante, tanto en lo que se refiere al proceso como al resultado final, $\mathrm{y}$, por otro lado, por la información que puede recoger el docente, que le puede permitir reorientar su enseñanza.

En la enseñanza por competencias la evaluación adopta un papel importante. Según las directrices del Espacio Europeo de Educación Superior, debe ser continua y formativa, con una gran diversidad de instrumentos y con el alumno como agente activo y, por tanto, evaluador (autoevaluación, coevaluación). Para que un estudiante adquiera destreza en una competencia, es necesario que conozca su descripción y los resultados de aprendizaje que se espera que alcance. Es por ello que los resultados de aprendizaje y los criterios de evaluación deben ser transparentes, explícitos, realistas y estar vinculados al proceso y al resultado final. Sólo así el alumno será 
consciente de qué se espera de él y podrá mostrarse responsable y autónomo en su desempeño.

En lo que se refiere a la evaluación del aprendizaje de la traducción, por tratarse de un saber procedimental, la evaluación sirve para comprobar si el alumno consigue resolver situaciones mediante la aplicación de los procedimientos en cuestión. Según sostiene Valls (1993: 190) se debería tener en cuenta que el estudiante posea el conocimiento suficiente sobre el procedimiento y sea capaz de usar y aplicar dicho conocimiento a situaciones concretas.

Para valorar la aplicación de los procedimientos se puede observar si el alumno realiza correctamente todos los pasos del procedimiento, solicitar que el alumno construya un diagrama de las acciones que ha seguido para la aplicación del procedimiento y valorar su adecuación, o se le puede pedir que explique en un informe cada paso que ha dado para ejecutar el procedimiento (cfr. infra 3.6).

En relación con la evaluación de la traducción, Kelly (2005: 131) apunta que a pesar de ser una práctica común entre los profesores de traducción, sólo cuenta con algunos estudios y reflexiones. El problema, según la autora, es que no se ha definido qué es una traducción aceptable y que la evaluación de la traducción se centra en el error, de modo que se restan puntos por cada error que comete un estudiante en una traducción y no se tienen en cuenta aspectos positivos, que quedan agrupados implícitamente en "no errores" y no tienen ningún impacto en la calificación. Además, las traducciones que se solicitan en las pruebas, a menudo no van acompañadas de una pequeña contextualización en forma de encargo de traducción.

\section{Propuesta pedagógica}

\subsection{Contextualización de la propuesta}

La propuesta que presentamos en este artículo se ha diseñado para la formación inicial de traductores del inglés al español, aunque podría servir para otras combinaciones lingüísticas.

Hemos partido de un curso de 150 horas de trabajo del estudiante, articuladas en 50 horas de trabajo presencial y 100 horas de 
actividades no presenciales. Durante las horas de trabajo no presencial se espera que el estudiante pueda hacer trabajo autónomo, en grupo o individual. Las sesiones de trabajo no presencial se distribuyen de acuerdo con los contenidos de aprendizaje. De este modo, se reservan para trabajar contenidos más conceptuales para los que se le pide al estudiante que lea material y que procese la información a su propio ritmo.

El objetivo general de la propuesta pedagógica para la formación inicial de traductores es familiarizar al estudiante con los aspectos generales de la traducción; se trata de la primera fase para la adquisición de la competencia traductora a que espera que llegue el estudiante al final de su formación. En este sentido, se tratan temas como la contrastividad entre las lenguas de trabajo, las herramientas del traductor, el proceso de documentación, las fases de un encargo de traducción o aspectos generales sobre el mercado laboral y se traducen textos sencillos de distintas tipologías textuales.

Las sesiones no presenciales se dedican a participar en el foro, o a realizar tareas de aprendizaje, en grupo o individualmente. Creemos que estas actividades promueven la autonomía del estudiante creando la posibilidad de que pueda adquirir información o conocimientos más conceptuales por su cuenta y, al mismo tiempo, un aprendizaje activo.

Para el buen desarrollo del curso, los estudiantes deben ser capaces de producir textos generales en su lengua materna $\mathrm{y}$ comprender textos generales en su lengua extranjera, además de utilizar las herramientas informáticas a nivel de usuario. Esto es necesario para que el estudiante se maneje con las herramientas de comunicación virtual, así como para que pueda traducir textos en diferentes formatos y soportes, y documentarse en la Red.

\subsection{Competencias}

Para diseñar la propuesta pedagógica hemos adoptado las competencias y los resultados de aprendizaje definidos por la Universitat Autònoma de Barcelona (UAB) para el Grado en Traducción e Interpretación de la Facultad de Traducción e Interpretación centro en el que hemos experimentado la propuesta. A continuación listamos las competencias específicas, con sus 
respectivos resultados de aprendizaje, que indican lo que el alumno ha de ser capaz de hacer:

i. Producir textos escritos en lengua materna para poder traducir: a) aplicar conocimientos léxicos, morfosintácticos y textuales; b) resolver interferencias de la combinación lingüística.

ii. Comprender textos escritos en idioma extranjero para poder traducir: a) aplicar conocimientos léxicos, morfosintácticos y textuales; b) aplicar estrategias para comprender textos escritos no especializados en lengua estándar; c) comprender la intención comunicativa y el sentido de textos escritos no especializados en lengua estándar.

iii. Utilizar los recursos tecnológicos para poder traducir: a) demostrar que se conocen los recursos tecnológicos básicos para poder editar textos escritos no especializados en lengua estándar.

iv. Utilizar los recursos de documentación para poder traducir: a) formular adecuadamente las necesidades informativas para poder traducir textos no especializados en lengua estándar; b) identificar las fuentes de información existentes (digitales y analógicas) para poder traducir textos escritos no especializados en lengua estándar; c) utilizar buscadores generales.

v. Dominar los principios metodológicos que rigen la traducción: a) identificar la traducción como un acto de comunicación dirigido a un destinatario; b) identificar el carácter textual y dinámico de la equivalencia traductora.

vi. Dominar los aspectos profesionales de la traducción: a) demostrar que se conocen las cuestiones básicas del mercado laboral de la traducción (ámbitos de empleabilidad, derechos y deberes del traductor).

vii. Resolver problemas de traducción de textos no especializados: a) identificar los problemas de traducción básicos de textos no especializados en lengua estándar; b) utilizar las estrategias y técnicas fundamentales para resolver problemas básicos de traducción de textos no especializados en lengua estándar. 
La única competencia transversal que se evalúa en este nivel es:

viii.Aprender de manera estratégica, autónoma y continua: a) formular un plan de acción para aprender.

\subsection{Contenidos de aprendizaje}

Los contenidos de aprendizaje son los siguientes:

ix. Principios metodológicos básicos que rigen la práctica de la traducción. Problemas, técnicas y estrategias fundamentales de la traducción de textos en lengua estándar

x. Resolución de dificultades contrastivas: diferencias de convenciones de la escritura, léxicas, morfosintácticas y textuales.

xi. Aspectos básicos del mercado laboral y del ejercicio de la profesión de traductor: ámbitos de empleabilidad; derechos y deberes del traductor.

xii. Uso de herramientas tecnológicas y de documentación básicas para la traducción de textos no especializados en lengua estándar.

\subsection{Secuenciación de las unidades didácticas}

Articulamos el proceso de enseñanza y de aprendizaje en unidades didácticas (cf. Galán-Mañas, 2009) (Tabla 1), que siguen una secuencia de dificultad creciente. Las tareas que conforman las unidades también siguen una progresión de dificultad: las tareas iniciales son simples y, a medida que el alumno va superando cada tarea, se introducen otras actividades más complejas, en las que se le piden más acciones y más relaciones con otros contenidos, y con conocimientos previos.

La primera unidad didáctica aproxima al alumno a los principios metodológicos de la traducción. En la primera tarea, el estudiante debe captar la importancia de la finalidad de la traducción y del destinatario, y practicar estrategias para comprender el texto original y reformularlo. En la segunda tarea, el estudiante debe comprender que la equivalencia traductora tiene un carácter dinámico, 
asimilar las diferencias fuera y dentro de contexto y practicar estrategias para la búsqueda de equivalencias contextuales. En la tercera tarea, el estudiante adquiere estrategias para captar el sentido de un texto. En la última tarea de esta unidad, el estudiante capta la necesidad de hacer frente a las interferencias de la lengua extranjera.

\section{Tabla 1. Unidades didácticas y tareas de traducción}

\section{\begin{tabular}{l|l} 
Unidades didácticas & Tareas de traducción
\end{tabular}}

1. Principios metodológicos de la traducción

Tarea 1. La finalidad comunicativa de la traducción

Tarea 2. El dinamismo de la equivalencia traductora.

Tarea 3. La importancia de la fase de comprensión.

Tarea 4. La importancia de la lengua de llegada

Tarea 1. Identificación de los aspectos contrastivos entre el inglés y el español.

2. Aspectos contrastivos entre la lengua extranjera y la lengua materna

3. La traducción de textos: narrativos, descriptivos, instructivos y argumentativos

Tarea final. Dossier de aspectos contrastivos entre el inglés y español

Tarea 1. La traducción de textos narrativos.

Tarea 2. La traducción de textos descriptivos.

Tarea 3. La traducción de textos instructivos.

Tarea 4. La traducción de textos argumentativos.

Tarea final. El encargo de traducción

Tarea 1. Las necesidades de documentación del traductor.

4. Recursos de documentación para traducir

Tarea 2. Los recursos electrónicos del traductor del inglés al español.

Tarea 3. La utilidad de los textos paralelos.

Tarea final. El itinerario de consultas en la búsqueda de información

Tarea 1. Salidas profesionales del traductor.

5. Introducción al mercado laboral de la traducción

Tarea 2. Obligaciones y derechos del traductor.

Tarea final. Debate en un foro sobre salidas profesionales, obligaciones y derechos del traductor. 
La unidad didáctica 2 está directamente relacionada con la anterior. En ella se profundiza en la necesidad de hacer frente a las interferencias de la lengua extranjera y se adquieren estrategias para evitar calcos. En la tarea final, los estudiantes realizan un trabajo escrito sobre los calcos, analizan una traducción en la que hay calcos, los clasifican, argumentan y proponen una solución para evitarlos.

En la unidad didáctica 3, empiezan a traducir textos sencillos de tipologías textuales diversas, en concreto textos narrativos, descriptivos, instructivos y argumentativos. En la tarea final, se les da un encargo de traducción y deben traducir un texto de una de estas tipologías de forma autónoma respetando el encargo.

La unidad didáctica 4 se centra en las herramientas del traductor de la combinación lingüística que nos concierne. En primer lugar, se les pide que identifiquen qué necesidades de documentación puede tener un traductor y que listen en qué fuentes pueden resolver dichas necesidades. En la tarea 2 se les pide que busquen y analicen diferentes recursos electrónicos y en papel (diccionarios monolingües, bilingües, listas de distribución y foros, traductores automáticos y libros de estilo). En la tercera tarea, deben reflexionar sobre la utilidad de los textos paralelos y dónde buscarlos. En la tarea final, los estudiantes ponen en práctica todo lo que han trabajado a lo largo de la unidad: se les ofrecen varios textos de diferentes temáticas y deben identificar los problemas que les plantean estos textos, documentarse para resolver dichos problemas, ya sea mediante recursos electrónicos o textos paralelos, y escribir un informe en el que argumenten todo el itinerario de consultas que han seguido.

La unidad didáctica 5 se realiza en forma de foro, en el que deben tratar las salidas profesionales del traductor y las obligaciones y derechos del traductor.

\subsection{Evaluación}

El sistema de evaluación de esta propuesta pedagógica contempla tres tipos de evaluación:

i. Evaluación diagnóstica, que se efectúa antes de iniciar el proceso de aprendizaje, con el fin de averiguar qué conocimientos tiene el alumno antes de iniciar el curso. Este tipo de evaluación no tiene repercusión en la calificación final. 
ii. Evaluación formativa, que verifica que el proceso de aprendizaje del alumno evoluciona de manera adecuada. No tiene ninguna repercusión en la calificación final de la asignatura y, de hecho, está presente en todas las actividades de aprendizaje, porque en todas ellas se recibe cierto tipo de retroalimentación.

iii. Evaluación sumativa, que determina la calificación final de la asignatura de cada estudiante.

A continuación detallamos cada una de ellas.

\subsubsection{Evaluación diagnóstica}

La evaluación diagnóstica se efectúa antes de iniciar el proceso de aprendizaje con el fin de averiguar qué conocimientos tiene el alumno antes de iniciar el curso. Lo ideal es que el alumno realice la misma prueba al finalizar el curso, de manera que se puedan cotejar los resultados y percibir su progreso.

Las preguntas diagnósticas pretenden recoger información sobre los conocimientos previos del alumno, sin la finalidad de calificarle; los comentarios en clase o en pequeños grupos de trabajo son también una base fundamental para conocer al grupo y a cada uno de sus miembros. La información que proporciona la evaluación diagnóstica determinará que el proceso de enseñanza-aprendizaje sea coherente con la situación inicial.

Para el diseño de la evaluación diagnóstica nos basamos en el cuestionario de Orozco y Hurtado (2002), aunque añadimos un apartado para recoger datos sobre el perfil del estudiante.

\subsubsection{Evaluación formativa}

El objetivo de las actividades es promover situaciones en las que los alumnos, en primer lugar, adquieran los conocimientos necesarios referidos a las competencias que van a desarrollar y, posteriormente, usen y apliquen dichos conocimientos en situaciones particulares. De hecho, tods las actividades del curso configuran la evaluación formativa, por recibir en todas ellas cierto tipo de retroalimentación. Las actividades son suficientemente diversas para 
promover distintos tipos de aprendizaje. Se espera que el alumno actúe, es decir, que realice las tareas que se le piden, pero también que reflexione sobre ellas, que planifique, revise, discuta, evalúe y se autoevalúe y que, en el caso de que sea necesario, sea capaz de buscar maneras de mejorar.

La mayoría de actividades va acompañada de una rúbrica de corrección. Estas rúbricas sirven para orientar al estudiante en la realización de la actividad, y que conozca de antemano qué se espera de él. Las rúbricas promueven también una evaluación más objetiva y sirven de instrumento de autoevaluación. Para la corrección de traducciones se utiliza un baremo de corrección de traducciones basado en Hurtado (1999), con el que se penalizan los errores pero también se premian los aciertos.

Algunas de las actividades que se realizan en el marco de la evaluación formativa son:

i. Identificar las características de los tipos textuales y compararlas en inglés y en español.

ii. Recoger y analizar textos paralelos y justificar su utilidad.

iii. Buscar y analizar herramientas electrónicas para el traductor y justificar su idoneidad en la traducción de textos.

iv. Debatir sobre los derechos y deberes del traductor.

v. Debatir las salidas profesionales de un traductor y las tareas que puede desempeñar.

vi. Definir un encargo de traducción.

vii. Justificar qué cambios puede sufrir una traducción que se ha hecho pensando en un encargo concreto si hubiera que adaptarla a un nuevo encargo.

viii. Documentarse para encontrar información y hacer un informe crítico sobre la información encontrada y su utilidad para un encargo de traducción concreto.

ix. Identificar y analizar los rasgos contrastivos entre la lengua extranjera y la lengua materna.

x. Realizar informes de traducción especificando el proceso que han seguido para elaborar la traducción.

xi. Realizar un análisis lingüístico, textual y extratextual de un texto antes de traducirlo. 
xii. Reconocer los mecanismos de coherencia y cohesión en los distintos tipos textuales.

xiii. Visitar una agencia de traducción y escribir un informe sobre las tareas que desempeña el personal, el tipo de encargos que reciben, programas informáticos que utilizan, tarifas que aplican, perfil de sus clientes, etc.

\subsubsection{Evaluación sumativa}

En la tabla 2 indicamos las actividades que forman parte de la evaluación sumativa con el porcentaje que les corresponde de la calificación total de la asignatura.

Tabla 2. Actividades de la evaluación sumativa

\begin{tabular}{l|c} 
Actividades & Porcentaje \\
Trabajo en grupo sobre aspectos contrastivos & $20 \%$ \\
\hline Traducción sintética & $15 \%$ \\
\hline Traducción obligatoria & $15 \%$ \\
\hline Prueba de traducción & $25 \%$ \\
\hline Foro (debate sobre mercado laboral) & $10 \%$ \\
\hline Carpeta del estudiante: & $15 \%$ \\
\hline
\end{tabular}

A continuación especificamos en qué consisten las actividades de la evaluación sumativa.

\subsubsection{Trabajo en grupo sobre aspectos contrastivos}

El alumno ha de realizar un trabajo en un grupo de tres personas, que consiste en recoger de distintas fuentes cuáles son los aspectos contrastivos más comunes entre la lengua extranjera y la materna. En concreto, hay que dividir los calcos en: calcos morfosintácticos, léxicos, de puntuación y otros (tipografía, convenciones, pesos y medidas, etc.). Explicar por qué se produce ese calco y añadir un ejemplo en el que se vea claramente el calco producido. Este apartado teórico se ha de acompañar de un texto traducido de la lengua extranjera a la lengua materna, en el que se hayan producido calcos y en el que los estudiantes deben indicar los calcos que encuentren, clasificarlos, proponer una corrección y argumentarla. 


\subsubsection{Traducción sintética:}

Esta actividad consiste en traducir un texto en lengua extranjera de forma resumida a la lengua materna, es decir, que la traducción tiene que sintetizar los puntos esenciales del texto original a modo de traducción resumida.

\subsubsection{Traducción obligatoria y prueba de traducción:}

La prueba consiste en la traducción individual de un texto general (aprox. 200 palabras) de la lengua extranjera al español. Los estudiantes pueden llevar toda documentación que consideren necesaria (diccionarios, apuntes, etc.) y recibirán tanto el texto que deben traducir como el encargo de traducción. Podrán añadir, si quieren, cualquier nota explicativa, destinada al cliente de la traducción. Esta prueba se realiza en aula multimedia, de modo que tienen acceso a cualquier recurso en línea que consideren necesario.

La traducción obligatoria tiene las mismas características que la prueba de traducción con la única diferencia de que la realizan en casa y tienen varios días para ello. Esta traducción deberá ir acompañada de un informe en el que se indiquen los pasos que se han seguido para hacer la traducción, los problemas que se han encontrado y cómo se han intentado resolver.

\subsubsection{Foro (debate sobre mercado laboral):}

En esta actividad, se propone a los estudiantes que participen en un debate en un foro. Para ello se divide la clase en grupos de 10 individuos, de modo que todos tengan la oportunidad de aportar ideas nuevas, algo más difícil en grandes grupos. En este foro se abren dos hilos temáticos: los ámbitos de empleabilidad del traductor $\mathrm{e}$ intérprete, y los derechos y deberes del traductor.

Para esta actividad, se exige al estudiante que haga, como mínimo, dos intervenciones en cualquiera de los dos hilos temáticos. Las intervenciones, para ser puntuadas positivamente, deben ser argumentadas y tener coherencia con las intervenciones anteriores. Una vez cerrado el foro, el estudiante debe escribir un informe individual de las principales ideas que han surgido en los distintos hilos temáticos del foro de cada uno de los grupos. 
3.5.3.5. Carpeta del estudiante:

La carpeta de aprendizaje del estudiante consiste en la recopilación de un conjunto de actividades que el estudiante ha llevado a cabo a lo largo del curso. El objetivo de la carpeta es explicar los progresos que ha hecho a lo largo de este período lectivo. Entre el material que debe entregar, se encuentran las versiones mejoradas de la traducción sintética y la traducción obligatoria, un resumen de dos conferencias relacionadas con la traducción a las que haya asistido el estudiante a lo largo del curso, reflexión sobre el proceso de aprendizaje de cada una de las unidades didácticas, tres actividades que demuestren la progresión en el proceso de aprendizaje, elegidas por el estudiante. Para orientar esta reflexión, se le proponían los siguientes enunciados: 1) lo que mejor ha entendido en esta asignatura, 2) lo que no le ha quedado claro de esta asignatura, 3) su plan de trabajo para mejorar en los aspectos que no le han quedado claros de asignatura (objetivos y plazos), 4) para qué le han servido las sesiones presenciales de esta asignatura y 5) para qué le han servido las actividades no presenciales de esta asignatura.

\section{Experimentación de la propuesta}

\subsection{Contexto de la experimentación y caracterización de la muestra}

La propuesta que presentamos se ha experimentado en la asignatura de Iniciación a la traducción del Grado en Traducción e Interpretación de la UAB a lo largo de dos cursos.

Los estudiantes que han participado han sido 32 el primer año y 34 el segundo. Todos ellos eran estudiantes que cursaban el primer año del Grado y no procedían de otros estudios superiores.

\subsection{Instrumentos para la recogida de datos}

\subsubsection{Cuestionario de evaluación diagnóstica}

La evaluación diagnóstica se realizó antes de iniciar el proceso de aprendizaje para identificar el nivel de los alumnos antes de 
empezar el curso, y se repitió al concluir el curso para que pudieran valorar los propios alumnos el progreso que habían hecho.

\subsubsection{Calificaciones de las actividades de aprendizaje}

Se han tenido en cuenta las calificaciones otorgadas a los estudiantes por parte del profesor en las actividades de las unidades didácticas que formaban parte de la evaluación sumativa. Cada actividad se ha corregido con una rúbrica en la que se indicaban los diferentes niveles de logro a que podía llegar el alumno. Estas rúbricas se ponían a disposición del alumno desde el inicio de curso para que tuviera conciencia de qué se esperaba de él. Además, permitían que la corrección del profesor fuera objetiva.

\subsubsection{Diario reflexivo del estudiante}

Al finalizar la asignatura, los estudiantes debían escribir un diario reflexivo en el que se les pedía que especificaran: 1) lo que mejor habían entendido de la asignatura; 2) lo que no habían entendido de la asignatura; 3) un plan de trabajo para mejorar los aspectos que no le hayan quedado claros a lo largo de la asignatura; 4) observaciones varias.

\subsubsection{Cuestionario de evaluación de la semipresencialidad}

Al finalizar el curso, los estudiantes también debían valorar la utilidad del modelo de presencialidad seguido. Para ello debían responder dos preguntas: 1) ¿para qué han sido útiles las sesiones presenciales; 2) ¿para qué han sido útiles las sesiones no presenciales?

\subsubsection{Diario reflexivo del profesor}

A lo largo de todo el curso, el profesor ha escrito un diario reflexivo en el que ha hecho constar observaciones de la progresión de las unidades didácticas. En el diario se han indicado las reacciones tanto de los alumnos como del profesor ante las distintas actividades, las dificultades que han surgido y otras cuestiones pedagógicas. 


\subsection{Resultados}

En este apartado presentamos el análisis de los resultados obtenidos.

4.3.1. Resultados de las calificaciones de las actividades de aprendizaje

Para los resultados de aprendizaje se han tenido en cuenta únicamente las calificaciones de la evaluación sumativa que presentamos separadas por curso. Las calificaciones oscilan entre total 0 y 10 , siendo que para aprobar se debe superar el 5 (cuadro 1).

Cuadro 1. Calificaciones de la evaluación sumativa

\begin{tabular}{|l|l|l|l|l|l|}
\multicolumn{1}{c}{} & $\begin{array}{l}\text { No } \\
\text { presentado }\end{array}$ & Suspenso & Aprobado & Notable & Sobresaliente \\
\hline 1er año & 12,50 & 12,50 & 28,12 & 31,25 & 15,62 \\
\hline $2^{\circ}$ año & 14,71 & 5,88 & 20,59 & 44,12 & 14,71 \\
\hline
\end{tabular}

A lo largo de los dos años, se puede comprobar que el porcentaje de estudiantes matriculados que no se presentaron a la evaluación final fue de 12,5 y 14,7. Este porcentaje tan alto podría deberse al aumento de los precios de matrícula en la universidad: un suspenso implica matricular la asignatura de nuevo por una tasa muy superior.

El porcentaje de suspensos es bastante bajo, mientras que el grueso de los estudiantes se sitúa entre el aprobado y el notable. El bajo porcentaje de suspensos podría deberse a que: 1) los estudiantes no que no se sienten seguros y piensan que van a suspender abandonan la asignatura para no tener que pagar las tasas de nuevo, 2) la diversidad de actividades y la evaluación continua promueven un aprendizaje progresivo $\mathrm{y}$, en consecuencia, aumenta el número de aprobados. Por otro lado, el grueso de los alumnos se concentra entre el aprobado y el notable, mientras que el porcentaje de sobresalientes es relativamente bajo. 


\subsubsection{Resultados del diario reflexivo del estudiante}

En el diario reflexivo, los estudiantes han señalado que al iniciar la asignatura tuvieron que hacer un gran esfuerzo de organización y gestión del tiempo, así como de responsabilidad para llevar las actividades al día. También han hecho constar que, a pesar de las reticencias iniciales para participar en el foro, al final, al ver los resultados del debate, les ha resultado muy útil para su aprendizaje. Muchos han destacado también que las actividades no eran rutinarias.

Según los comentarios de los estudiantes, la carpeta de aprendizaje ha favorecido que reflexionaran sobre su proceso de aprendizaje, pero también para trazar un plan de trabajo con el que mejorar los aspectos que no les habían quedado claros durante el curso.

Este diario nos ha servido para conocer la opinión de los estudiantes sobre el desarrollo de la asignatura y detectar si había algún aspecto que, de forma generalizada, no había quedado claro.

Las respuestas a las preguntas que se les hacía, se pueden resumir en:

i. Lo que mejor han entendido de la asignatura:

- Ser metódicos en sus traducciones.

Hacer un preanálisis de los textos para comprenderlos mejor.

Consultar fuentes de documentación muy diversas.

Saber que existen muchas especialidades de traducción.

Los derechos y obligaciones del traductor.

El dinamismo de la equivalencia traductora.

Los aspectos contrastivos entre las lenguas de trabajo.

Traducir no significa ir palabra por palabra.

ii. Lo que no han entendido:

La traducción de referentes culturales.

Algunos estudiantes han tenido dificultades en la traducción sintética.

iii. Plan de trabajo para mejorar los aspectos que no me han quedado claros:

Practicar con traducciones.

Leer más inglés para ganar fluidez en las traducciones. 
Revisar los manuales de estilo, especialmente para repasar los calcos tipográficos del inglés.

\subsubsection{Resultados de la evaluación de la semipresencialidad}

El cuestionario ha permitido constatar la utilidad de las sesiones no presenciales; durante estas sesiones los estudiantes han realizado actividades que promueven el aprendizaje autónomo y la autorregulación.

Según los estudiantes, las sesiones presenciales han sido útiles para:

i. Aprender a traducir.

ii. Conocer cómo funciona el mundo laboral de los traductores.

iii. Aclarar errores.

iv. Adquirir una dinámica para traducir.

v. Aclarar dudas con la profesora y contrastar actividades con los compañeros

Las sesiones no presenciales, por otro lado, les han sido útiles para:

i. Asistir a conferencias.

ii. Participar en el foro.

iii. Reflexionar y avanzar en los trabajos de equipo que había que entregar.

iv. Documentarse por su cuenta.

v. Consultar dudas con la profesora en su despacho.

4.3.4. Resultados del diario reflexivo del profesor

Las observaciones del profesor en el diario reflexivo han sido:

4.3.4.1. Cuestiones relacionadas con los estudiantes

i. Al principio de la asignatura, los estudiantes se sentían algo inseguros ante la idea de tener algunas sesiones no presenciales en las que deberían realizar actividades con herramientas con las que estaban poco familiarizados, como el foro, el blog o el wiki.

ii. Se observa que, como los estudiantes han cursado la asignatura Introducción la informática para traductores durante 
el primer semestre, no tienen problemas con el uso de las herramientas electrónicas en general.

iii. El primer año que se implantó esta propuesta, los grupos de trabajo los creó el docente aleatoriamente. Esto provocó muchos problemas internos y fue una queja generalizada por parte de los estudiantes. El segundo año se dejó a los estudiantes que crearan los grupos ellos mismos, y sólo hubo problemas en un grupo debido a que se había creado con las personas que quedaban sueltas y no habían formado grupo.

iv. La mayoría de estudiantes no participa en aquellas actividades que no puntúan. Por ejemplo, se les pidió que realizaran una actividad en un wiki y sólo cuatro personas participaron en ella. Ninguno de los estudiantes había trabajado con esta herramienta anteriormente.

v. En lo que respecta a la evaluación, en el primer año de implantación los estudiantes se quejaron de que: tenían muchos entregables que puntuaban y que debían controlar muchas fechas diferentes; necesitaban más tiempo para algunas de las actividades que puntuaban; consideraban que el trabajo de aspectos contrastivos había requerido un gran esfuerzo y propusieron que en vez de que representara el $15 \%$ de la nota total de la asignatura, lo hiciera con un $20 \%$.

\subsubsection{Cuestiones técnicas}

El centro educativo no dispone de suficientes aulas equipadas con ordenadores en las que los estudiantes puedan trabajar en grupo durante las sesiones no presenciales. Ello hizo necesario abrir el aula en la que habitualmente hacíamos clase para que pudieran trabajar durante las sesiones no presenciales.

\subsubsection{Cuestiones relacionadas con el profesor}

El primer año se observó que era necesario reducir la carga de trabajo del profesor y de los estudiantes, a los que se les pedían muchos entregables. En el segundo año, la carga de trabajo del estudiante se redujo y, en consecuencia, la del docente también. 
Este diario nos ha servido para revisar el diseño de las tareas y revisar la evaluación. También ha servido para comprobar que las sesiones no presenciales realmente promueven la autonomía del estudiante.

\subsection{Consideraciones sobre la experimentación de la propuesta}

Siguiendo las sugerencias de los estudiantes, tras el primer año de experimentación de la propuesta tuvimos que introducir algunos cambios, como una mejor explicitación de las consignas, el cambio de algunos de los textos de trabajo, la modificación de algunos porcentajes en la evaluación sumativa, la asignación de más tiempo para algunas actividades.

Creemos que la modificación de algunos de estos aspectos ha tenido una repercusión positiva tanto en la carga de trabajo del estudiante como del profesor. En lo que respecta al profesor porque se ha reducido el número de entregas, han disminuido también las entregas calificables y porque algunas actividades han pasado a formar parte de la coevaluación y las han corregido los compañeros. Para el estudiante también ha sido positivo poder realizar las actividades sin la presión de una calificación.

Según han hecho constar en su diario de aprendizaje, observamos que están satisfechos con la metodología utilizada y que valoran positivamente la diversidad de actividades de aprendizaje y el hecho de disponer de algunas sesiones no presenciales para poder realizar ciertas actividades.

\section{Conclusiones}

La propuesta pedagógica para la formación inicial del traductor que acabamos de presentar tiene en cuenta los diferentes elementos del diseño curricular: la determinación de las competencias que se pretenden desarrollar y evaluar, los materiales y recursos, los indicadores de lo que se espera que es una demostración de la competencia. 
Las actividades de aprendizaje, que se han diseñado para que sean variadas, se han creado en función de la competencia que se deseaba desarrollar, la metodología que mejor se ajustaba para adquirir dicha competencia, el tipo de contenidos asociados a la actividad (conceptuales o procedimentales), la interacción de la actividad (individual o en grupo), el grado de presencialidad y no presencialidad, el tiempo necesario para la ejecución de la actividad, las herramientas que mejor se adapten a la actividad y de qué forma se evaluará la actividad.

Con la selección de herramientas tecnológicas para realizar ciertas actividades pretendíamos crear un espacio de comunicación asíncrona y sincrónica que complementara la comunicación presencial y en el que se pudieran desarrollar actividades formativas, además de servir como repositorio de materiales de aprendizaje. Para ello, las herramientas que hemos propuesto son: el entorno virtual de aprendizaje, el foro, el correo electrónico, el uso compartido de documentos, el chat de voz y de texto, el blog y el wiki.

Además, hemos evaluado no sólo el producto final sino también el proceso; hemos dado a conocer los criterios de corrección y calificación de todas las actividades, especialmente de las actividades que forman parte de la evaluación sumativa; hemos penalizado los errores pero también hemos premiado los aciertos; hemos acompañado los textos que debían traducir los estudiantes de un encargo que contextualizara la tarea o bien hemos solicitado que fueran ellos mismos los que definieran el encargo.

En este sentido, la evaluación es una evaluación para el aprendizaje y no del aprendizaje, y sirve para comprobar si se han adquirido las competencias y en qué grado, proporcionar información sobre su progreso, sus errores, sus aciertos y posibles maneras de mejorar.

Por último, destacamos que el sistema de evaluación diseñado garantiza la diversidad de actividades evaluadoras con el objetivo de cubrir los diferentes estilos de aprendizaje de los estudiantes.

Consideramos que esta propuesta se puede adaptar a cualquier combinación linguística y con ella pretendemos hacer una pequeña aportación a la didáctica de la traducción dada la escasez de diseños curriculares existentes para la formación de traductores, especialmente 
cuando se trata de propuestas basadas en la formación por competencias.

\section{Bibliografía}

CALVO, Elisa. Translation and/or translator skills as organising principles for curriculum development practice. Jostrans, v. 16, p. 5$25,2011$.

DÍAZ FOUCES, Óscar. Didáctica de la traducción:PortuguésEspañol. Vigo: Servicio de Publicación da Universidade de Vigo, 1999.

GALÁN-MAÑAS, Anabel; HURTADO, Amparo. Blended learning in translator training: methodology and results of an empiric validation. The Translator and Interpreter Trainer, v. 4, n. 2, p. 197-231, 2010.

GALÁN-MAÑAS, Anabel. La enseñanza de la traducción en la modalidad semipresencial. Departamento de Traducción e Interpretación. Tesis (Doctorado en Traducción e Interpretación) Universidad Autónoma de Barcelona, 2009.

GALÁN-MAÑAS, Anabel. La enseñanza por competencias, por tareas y por objetivos de aprendizaje: el caso de la traducción jurídica portugués-español. Ikala. Revista de Lenguaje y Cultura, v. 12, n. 18, p. 27-57, 2007.

GAMERO, Silvia. Traducción alemán-español: aprendizaje activo de destrezas básicas. Castellón de la Plana: Servei de Publicacions de la Universitat Jaume I, 2005.

GONZÁLEZ DAVIES, Maria. Multiple voices in the translation classroom: activities, tasks, and projects. Amsterdam, Philadelphia: J. Benjamins Publications, 2004.

. (Coord.). Secuencias: tareas para el aprendizaje interactivo de la traducción especializada. Barcelona: Octaedro, 2003. 
HURTADO, Amparo. Compétence en traduction et formation par competences. TTR, v. 21, n. 1, p. 17-64, 2008.

. Competence-based curriculum design for training translators. The Interpreter and Translator Trainer, v. 1, n. 2, p. 163-195, 2007.

. (Dir.). Enseñar a traducir. metodología en la formación de traductores e intérpretes. Col. Investigación Didáctica. Madrid: Edelsa, 1999.

. La enseñanza de la traducción directa general. objetivos de aprendizaje y metodología. In: HURTADO, Amparo. (Ed.). La enseñanza de la traducción. Castellón de la Plana: Servei de Publicacions de la Universitat Jaume I, 1996.

- Didactique de la traduction des textes specialisés. In: 3ème JOURNÉE ERLA-GLAT. LEXIQUE SPECIALISÉ ET DIDACTIQUE DES LANGUES, 1992, Brest. Actes ... Brest: UBO-ENST, 1992. p. 921.

KELLY, Dorothy. A handbook for translator trainers. Manchester: St. Jerome, 2005.

OROZCO, Mariana. Traducción del inglés al castellano. Bellaterra: Servei de Publicacions de la Universitat Autònoma de Barcelona, 2007.

PACTE. Investigating translation competence: conceptual and methodological issues. Meta, v. 50, n. 2, p. 609-619, 2005.

SEVILLA MUÑOZ, Manuel. Didáctica de la traducción científicotécnica (inglés-español) para estudiantes de humanidades. Tesis (Doctorado) - Universidad Complutense de Madrid, 2002. 
VALLS, Enric. Los procedimientos: aprendizaje, enseñanza y evaluación. Barcelona: ICE-Universitat de Barcelona y Editorial Horsori, 1993.

VILLA, Aurelio; POBLETE, Manuel (Dirs.). Aprendizaje basado en competencias: una propuesta para la evaluación de las competencias genéricas. Bilbao: Universidad de Deusto, 2007.

ZABALA, Antoni; ARNAU, Laia. 11 ideas clave:cómo aprender y enseñar competencias. Barcelona: Ed. Graó, 2007.

Submetido em: 13/12/2013 Aceito em: 25/11/2014

Título: Ensino da iniciação à tradução baseado em competências: resultados de um estudo empírico

Title: Teaching introduction to translation in a competence-based approach: results of an empirical study 